Agosto 2012 | www.ocula.it - DOI: 10.12977/ocula15

\title{
Dare forma allo spazio del convivere
}

\author{
di Anna Lazzarini
}

Università di Bergamo-anna.lazzarini@unibg.it

\begin{abstract}
Today, the relationship between social, cultural, political transformations and spatial processes can be critically rethought as the increasing articulation of spaces of places and spaces of flows.

This article argues that the relationship between architecture and politics emerges through the intertwining of issues present in the discourses and the practices of both, which entails a prospective of action and transformation of the city that necessarily expresses an assumption of responsibility. These issues link the city's historicalcultural and material-functional dimensions and originate from memories and the possibility of their future destination. They refer also to the ability to perceive, experience, and enact alternatives to the present: they concern the imaginative ability to think otherwise.

In this regard, the multidimensional notion of "public space" is crucial, because it involves the articulation of spaces as well as the function and significance of buildings, but also the quality of relationships, experiences and urban life that happen in them.

The weaving of flows, networks, places, and bodies in the urban fabric promises new forms of being-in-common that invite architecture, urban planning, and politics to be interpreters of this "desire for connection", the peculiar aspect that defines spatial and symbolical forms, but also relational modalities.
\end{abstract}

\section{Keywords}

flows/places; responsibility; architectural project; public space.

\section{Sommario}

1. La rivoluzione dello spazio globale

2. Responsabilità. La città fra memoria e destinazione

3. Progetto. Uno sguardo rinnovato sulla città

4. Ripensare lo spazio pubblico

Bibliografia 


\section{Ocula ${ }^{13}$}

Architettura e politica: un incrocio di sguardi | Anna Lazzarini, Dare forma allo spazio del convivere.

\section{La rivoluzione dello spazio globale}

Molteplici sono le tensioni che prendono corpo entro la città e si addensano in particolare attorno al nodo del rapporto fra politica e architettura: "un incrocio di sguardi" fra queste prospettive consente di interrogarsi sul legame tra forme e dinamiche spaziali e trasformazioni sociali, culturali, politiche, sapendo che tali trasformazioni oggi riguardano la crescente articolazione fra spazio dei luoghi e spazio dei flussi.

Le relazioni fra le forme architettoniche e insediative e le caratteristiche proprie delle formazioni sociali, che in ogni epoca mai si presentano come lineari, nella contemporaneità evidenziano tratti di grande criticità e interesse, e rendono il nesso fra configurazioni e funzioni sempre più problematico. È questa una delle conseguenze della «rivoluzione dello spazio» (cfr. Castells 1989: 43, tr. it.), una dimensione fondamentale del processo di trasformazione strutturale che sta interessando la società contemporanea in tutte le sue articolazioni. Per questo motivo sembra necessario concepire una nuova teoria delle forme e delle dinamiche spaziali, che si mostri conforme al contesto economico, tecnologico, sociale e culturale. È dunque possibile guardare alle città e alle loro recenti trasformazioni come all'epicentro dei mutamenti dei sistemi socio-spaziali di comunicazione culturale.

La globalizzazione si configura come un processo planetario di despazializzazione e di rispazializzazione: una nuova logica di articolazione dello spazio e del tempo determina una profonda riorganizzazione della vita sociale. Ciò che cambia non è solo la geografia politica ed economica, ma è la natura stessa dei luoghi e delle loro relazioni. I flussi di merci, capitali, persone, informazioni, simboli generano una nuova «metageografia», meno dipendente dalle formazioni territoriali che hanno preso forma durante la supremazia dello stato nazionale (cfr. Magatti 2005: 19). L'infrastruttura fondamentale attorno alla quale si sviluppa questa nuova «metageografia» è costituita dalla rete globale. I nodi di questa rete concentrano le funzioni strategiche e direttive del capitalismo, attivando relazioni e scambi che sono parzialmente indipendenti dai vincoli territoriali e dai confini fra gli stati, almeno per ciò che riguarda aree che appaiono politicamente omogenee.

La rete oggi costituisce, secondo Manuel Castells, la nuova morfologia sociale (cfr. Castells 1996): la diffusione della logica di rete modifica in modo strutturale le modalità e i risultati dei processi di produzione, di diffusione e di scambio, come anche le forme dell'esperienza, del potere e della cultura stessa. Per la verità, la forma a rete dell'organizzazione sociale è sempre esistita, ma gli sviluppi delle tecnologie dell'informazione permettono la sua espansione pervasiva attraverso l'intera struttura sociale.

Tuttavia, anche nel contesto globale, il territorio svolge un ruolo specifico non eludibile, in particolare proprio nelle realtà urbane. Infatti, la proliferazione delle reti globali e le nuove forme di digitalizzazione di ampie varietà di attività economiche e politiche favoriscono lo sviluppo di nuove scalarità: l'ascesa di scale subnazionali come le città globali, e di scale 


\section{Ocula ${ }^{13}$}

Architettura e politica: un incrocio di sguardi | Anna Lazzarini, Dare forma allo spazio del convivere.

sovranazionali, come i mercati globali. Per questo occorre interrogarsi circa l'impatto che questi nuovi sviluppi hanno sui territori, o meglio sui luoghi.

L'attuale rivoluzione socio-spaziale si rende visibile in modo esemplare negli spazi urbani e presenta alcune caratteristiche che occorre prendere in considerazione, non solo per comprenderne la complessità, ma anche per affrontare la sfida del loro governo.

La trasformazione della città nell'era dell'informazione si organizza lungo alcune direttrici bipolari e tensioni, la più significativa delle quali riguarda le forme dello spazio. Proprio nella realtà urbana il tema delle forme spaziali permette di interpretare le trasformazioni sociali, culturali, politiche del nostro tempo e di attribuirle essenzialmente alla crescente tensione fra spazio fisico e spazio dei flussi. Questi nel loro intreccio stabiliscono inediti collegamenti fra luoghi fisicamente separati (creati da un network interattivo di relazioni fra attività e individui che prescinde dallo specifico contesto di riferimento) e lo spazio fisico costruito (che organizza le esperienze umane negli ambiti della collocazione geografica).

La metropoli non si annulla nelle reti virtuali, ma si trasforma attraverso l'interrelazione fra comunicazione elettronica e relazioni fisiche, fra reti e luoghi.

\subsection{Abitare tra flussi e luoghi}

Lo spazio dei flussi è radicato nello spazio fisico, ma le loro logiche sono profondamente differenti. Il problema - teorico, sociale e politico, ad un tempo - è dunque quello di garantire un'adeguata articolazione fra i due sistemi: nella società delle reti emergono nuove forme e relazioni spaziali, che rendono necessario un ripensamento delle forme dell'architettura, della teoria urbanistica, ma anche della stessa teoria politica.

Il nuovo sistema di comunicazione e di informazione permette la crescente dissociazione fra prossimità spaziale e svolgimento delle funzioni quotidiane (lavoro, shopping, divertimenti, servizi pubblici...). Si tratta forse della fine della città quale spazio di concentrazione delle funzioni e dei luoghi simbolici che le incarnano? Il legame tra networks e località deve essere compreso a un livello più profondo, come geometria variabile che si realizza tra digitale e non digitale, tra flussi e luoghi. In questo modo, possiamo considerare spazi, ingressi e corridoi di connessione attraversati da flussi migratori internazionali, turisti, pendolari, come punti di passaggio della socialità. L'identificazione culturale e personale con questi posti, la loro funzionalità, i loro simbolismi diventano questioni sociali.

Il fattore critico che interessa i nuovi centri e i nuovi processi urbani riguarda il fatto che lo spazio appare sempre più differenziato in termini sociali, pur essendo profondamente interrelato in termini di funzioni, ben oltre la contiguità fisica. La conseguenza è proprio la relativa separazione fra il significato simbolico, la localizzazione delle funzioni e l'appropriazione sociale dello spazio nell'area metropolitana.

Ciò che contraddistingue i luoghi su cui le città si costruiscono e costruiscono la propria identità è la loro natura di spazi di significazione, la 


\section{Ocula ${ }^{13}$}

Architettura e politica: un incrocio di sguardi | Anna Lazzarini, Dare forma allo spazio del convivere.

loro capacità simbolica, la loro qualità antropologica: si tratta della straordinaria capacità di mettere in relazione spazi e forme di socialità, pietre, paesaggi e persone, persone fra loro, di intrecciare trame di significati, di simboli, di storie, di vite... I luoghi, definiti dalle cornici della memoria e della sedentarietà, rimangono ancora importanti per la vita sociale poiché in essi si esprimono aspetti irrinunciabili dell'esperienza umana, quali la riproduzione fisica e familiare, le relazioni interpersonali, la struttura spaziale degli artefatti culturali. Tuttavia, è innegabile che i loro referenti funzionali, simbolici, culturali e sociali siano sempre più intrecciati e in parte condizionati dalla logica dei flussi (ivi: 45 tr. it.).

Fra la logica dei flussi e quella dei luoghi si determina una tensione che si scarica sulla vita della città in una pluralità di modi. Occorre innanzitutto evitare un confronto in termini di contrapposizione tra le due dimensioni spaziali sulla base di un'aprioristica attribuzione di valore o di disvalore. Le relazioni spaziali, sia nel caso dei flussi sia in quello dei luoghi, sono contraddistinte contemporaneamente da forme di individualismo e di comunitarismo, da chiusure e da aperture, da dinamiche che favoriscono e che ostacolano la socializzazione.

Per quanto riguarda i flussi, le élite ne orientano le scelte e ne gestiscono processi, poteri e profitti. Esse costituiscono una realtà specifica e separata rispetto alla maggioranza delle persone che "utilizza" i flussi, ma che è estranea al loro controllo e alla determinazione delle strategie che li riguardano. Tuttavia, i flussi continuano a rappresentare movimenti, passaggi, trasferimenti da un luogo a un altro luogo: la mobilità del mondo contemporaneo, per quanto fluida o liquida possa essere pensata e rappresentata, non può essere considerata in antitesi all'esistenza dei luoghi, poiché tale mobilità prende corpo e accade proprio dentro i luoghi. Non si tratta di due dimensioni spaziali contrapposte e reciprocamente escludentesi, ma di un processo dinamico e complesso che si realizza entro uno spazio che ha assunto la forma della rete.

Lo spazio dei luoghi e lo spazio dei flussi non si escludono, ma coesistono, sia pure con modalità assai diverse rispetto al passato. Infatti, da sempre la territorialità ha cercato di controllare i flussi, ma al contempo, anche il carattere dei luoghi è sempre stato plasmato dagli innumerevoli scambi con l'esterno: il legame con il luogo è oggi in misura crescente iscritto nell'ipermobilità di alcuni suoi componenti, prodotti o risultati. Sono entrambi parti di un medesimo processo: il loro rapporto è identificabile con quanto Saskia Sassen chiama «embricazione» (cfr. Sassen 2006), quella relazione che consiste nella reciproca implicazione, o co-implicazione, ma anche nella necessaria e reciproca irriducibilità.

Se la città si configura come quello spazio privilegiato di intersezione fra locale e globale - fra logica dei luoghi e logica dei flussi - occorre provvedere a un ripensamento radicale di questa relazione, proprio entro l'idea di una "città delle reti". "Locale" non è il confinamento entro un ambito ristretto, ma è sempre oltre i propri confini: ciò che è locale è già da sempre in grado di ospitare ciò che è globale. È il "locale" che riceve su di sé flussi di migranti, di pendolari, di turisti; è il "locale" che modifica profondamente il proprio aspetto fisico sulla base dei flussi di informazioni, di simboli, di segni; è il 


\section{Ocula ${ }^{13}$}

Architettura e politica: un incrocio di sguardi | Anna Lazzarini, Dare forma allo spazio del convivere.

"locale" lo spazio su cui sorgono imprese transnazionali, che parla molte lingue e che diventa spazio conteso nei conflitti che la molteplicità di codici, interessi, visioni produce.

Così la città subisce e contemporaneamente attiva un duplice movimento: da un lato si ri-centra, vivifica il suo "centro" - nel senso di ciò che sta al centro sul piano fisico, ma soprattutto di ciò che è centrale sul piano simbolico, cioè gli spazi pubblici e i luoghi dell'abitare -, riportandovi funzioni economiche, politiche o culturali, valorizzando i luoghi simbolici; dall'altro, attua un continuo processo di sconfinamento, poiché ogni nuovo progetto di città, dislocato entro confini amministrativi o fisici sempre meno definiti, diventa l'esito dell'incrocio di flussi materiali e immateriali (cfr. Perulli 2007: 55).

Ma come abitare i flussi? E come non restare intrappolati nelle angustie dei luoghi? Come programmare e gestire il loro intreccio nel modo più creativo per la città?

\section{Responsabilità. La città fra memoria e destinazione}

Queste domande richiedono di ripensare criticamente le relazioni fra architettura e politica a partire da alcune questioni che intessono il discorso e le pratiche di entrambe.

Innanzitutto entrambe riguardano una prospettiva di azione e di trasformazione della città che si traduce nell'assunzione di una responsabilità; intrecciano la dimensione storico-culturale e quella materiale, funzionale.

L'esperienza della città si dispiega nello spazio compreso fra un tessuto di memorie, di storie, di tradizioni che prendono corpo nei monumenti, e la possibilità di una proiezione nel futuro, la necessità di pensarsi continuamente entro una dimensione di ulteriore destinazione. E proprio in questo scarto fra ciò che è stata e ciò che sarà, fra la trama di significati che sono stati condivisi e il disegno che progetta di perseguire, si apre la dimensione più autenticamente politica della città.

Parlare di memoria e destinazione significa fare riferimento a tessuti di significati intrecciati, sovrapposti e in continuo mutamento in cui emergono l'identità della città e dei suoi abitanti. La città è dunque quel patrimonio immateriale che è combinazione di simboli, trama di relazioni che i cittadini intrecciano fra loro e con il loro passato. È valore iscritto in un'esperienza storica, per diventare "categoria politica". La città è una realtà storicopolitica di libertà, di simboli e di legami, che, se distrutta, non può più essere restaurata, poiché è intessuta della realtà vivente di relazioni, azioni, parole, memorie.

Ciò consente di leggere nella città quel tratto poetico e politico ad un tempo, che guarda al passato storico attraverso l'esercizio critico del pensiero, e al presente vivente come aperto a quanto è inatteso, incompiuto.

La città, dunque, in ogni epoca costringe a stabilire un rapporto con il passato. Il presente visibile e iscritto nel corpo della città sembra in grado di riassumere e condensare in sé tutto il corso del tempo. 


\section{Ocula ${ }^{13}$}

Architettura e politica: un incrocio di sguardi | Anna Lazzarini, Dare forma allo spazio del convivere.

Tale condensazione del tempo nel presente è un aspetto centrale nell'intreccio di esperienza individuale e collettiva, quotidiana e storica. Ed è il presente a garantire la qualità relazionale costitutiva della città, in quanto mette in relazione fra loro passato e futuro, memoria e progetto: il presente appare così in tutta la sua "densità", densità significante, narrativa, iconica, storica, assiologica...

Le forme di narrazione sulla e della città sono modalità privilegiate sia per intrecciare insieme le dimensioni del tempo, sia per evidenziare e risignificare la matrice relazionale della città, come trama di immagini, significati, prospettive... L'aspetto fisico della città, il suo corpo, appare come oggetto di un conflitto incessante di interpretazioni, riscritture materiali, riusi, restauri, cambiamenti di rappresentazione o di funzionalità delle stesse strutture.

La città è un serbatoio di conoscenza e di possibilità che nessuno può esaurire o organizzare definitivamente, un testo vivente che consente a ciascuno di costruirsi un proprio particolare itinerario conoscitivo e narrativo. Tuttavia, la città appare come un testo difficile da leggere per la complessità dei messaggi, la crescente polisemia delle parti, le diverse abilità e codici dei lettori, l'inevitabile commistione di memoria e leggenda.

Ma è soprattutto l'apertura al futuro a interrogare il rapporto fra architettura e politica: come rispondere dell'avvenire della città? E chi deve risponderne?

Qui si esprime la questione della responsabilità (cfr. Derrida 1985: 251, tr. it.) in tutta la sua consistenza politica, poiché riguarda sempre la relazione con l'altro, con gli altri, persino con l'alterità dell'avvenire. Progettare una città è pensarla come un luogo a venire, tuttavia un luogo a venire in un certo senso "prescritto" dalla sua stessa memoria, poiché ogni città è luogo di storia stratificata nelle sue mura, nei monumenti, nelle strade. Si tratta di una storia che resta a venire, poiché interroga circa ciò che si intende fare della città stessa e delle forme di convivenza dei suoi cittadini.

Per questo motivo non può darsi alcun progetto esaustivo, definitivo, "totalitario" di città: ogni progetto concernente il destino della città, ciò che lega la sua memoria al suo presente e al suo avvenire, supera infatti lo spazio di una generazione. Occorre, invece, dare tempo e spazio alle generazioni future e lasciare la città, intesa anche nella sua responsabilità etica e politica, come eredità da custodire e dono da trasformare.

Per esprimere in tutta la sua potenza simbolica la questione della responsabilità, della risposta da dare e da chiedere, del vincolo di una dimensione politica intesa come destinazione, Derrida mostra come la città si situi proprio fra memoria e promessa: «il tempo implica una promessa che impegna qui più di una generazione, e dunque più di una politica, più che la politica, in una durata la cui eterogeneità, perfino la discontinuità, la nontotalizzazione devono essere accettate come legge» (ivi: 255). 


\section{Ocula ${ }^{13}$}

Architettura e politica: un incrocio di sguardi | Anna Lazzarini, Dare forma allo spazio del convivere.

\section{Progetto. Uno sguardo rinnovato sulla città}

Anche l'idea di "progetto" deve essere oggetto di un riesame critico. Oggi la tradizione metodologica moderna in architettura, che prevede una concatenazione lineare fra intenzioni, piani, progetti e opere, non sembra più adeguata alle recenti trasformazioni e alla loro complessità. Il progetto non può essere dedotto da un sistema di regole e funzioni predefinite, né può rappresentare un disegno concepito a priori: esso emerge invece quale esito di una molteplicità di condizioni e potenzialità contestuali, attraverso processi plurali e articolati. Questo non significa evidentemente svilire intenzionalità e responsabilità di chi governa, ma concepire relazioni più complesse, oblique e indirette fra intenzionalità ed esiti, fra testo e contesto.

Un approccio epistemologico consente di individuare nel progetto una modalità attiva, interpretativa, trasformativa e complessa di guardare la città e di pensarne le forme.

Punto di partenza della riflessione è infatti la modalità stessa di guardare, poiché la strategia dello sguardo influenza in modo significativo la capacità di progettare la città. Lo sguardo progettuale è, infatti, caratterizzato da un deciso superamento della concezione rappresentazionale della realtà, come replica del mondo ontologicamente dato, in favore di un'istanza costruttiva che persegue la progettazione di mondi (cfr. Ceruti: 1989). Si tratta dunque di uno sguardo attivo che costituisce esso stesso un processo di costruzione della città. Dunque, uno sguardo interpretativo, intenzionale e rivolto al futuro: l'obiettivo è leggere nel tessuto della città le sue stesse potenzialità evolutive e autopoietiche. Si introduce in questo modo una prospettiva di azione e di trasformazione della città che può tradursi anche nell'assunzione coraggiosa di un punto di vista e di una responsabilità sociale e politica.

Inoltre, questo approccio, sul piano conoscitivo e tecnico, invita a superare alcune scelte ereditate dal contesto disciplinare, come l'approccio riduzionistico e non relazionale ai problemi della città e del suo territorio, l'amplificazione del ruolo e dei risultati delle tecniche, l'enfasi sull'applicazione settoriale, per compartimenti. Vengono in questo modo messi in discussione criticamente alcuni metodi di elaborazione dei progetti di trasformazione urbana e territoriale e la loro pretesa di autonomia. In questo senso, «il territorio si offre come "testo" per il ripensamento interpretativo, costruttivo, multidisciplinare del progetto» (Maciocco e Tagliagambe 1997: 196).

Ogni progetto in quanto tale si situa fra realtà e possibilità, fra persistenza e mutamento: si configura come la proposta di un diverso futuro che deve fare i conti con il fatto che, nel mentre essa si dispiega e prescrive i mezzi per cambiarlo, il mondo è già cambiato e che, gli strumenti e le modalità del mutamento vanno sottoposti a continua revisione sulla base delle modificazioni della realtà. Così, se il reale condiziona il possibile, è vero anche il contrario: progettare alternative al presente significa che quanto è accettato come ineluttabile e necessario, in realtà continua a essere aperto al cambiamento. 


\section{Ocula ${ }^{13}$}

Architettura e politica: un incrocio di sguardi | Anna Lazzarini, Dare forma allo spazio del convivere.

Un'interpretazione possibile del dialogo fra progetto e contesto permette di concepire il "progetto come una possibilità evolutiva virtuosa del contesto, capace di conciliare provvisoriamente visioni parziali e interesse collettivo, forme e significati, vecchi e nuovi valori» (Palermo 2007: 27).

Ma certo non sono solo le dimensioni dello spazio e del tempo nelle loro diverse articolazioni a costituire la sostanza della città. Ogni città, come insiste Calvino (cfr. Calvino 1972), è fatta anche delle percezioni, dei sogni, delle paure, delle illusioni, dei desideri di chi la abita... E le domande che ciascuno pone alla città (e viceversa che la città pone a ciascuno) e le risposte che riceve influenzano fino a determinarlo il modo di vivere la città medesima, la percezione della sua forma e della sua struttura.

Il progetto accade dunque fra effettualità e possibilità, entro le loro continue oscillazioni: si configura come l'integrazione di ciò che è dato, del dato di realtà, nel vasto orizzonte delle sue possibili trasformazioni. Esso rimanda a possibilità di percepire, esperire, pensare e agire alternative rispetto a quelle attuali e operanti.

Tale attitudine di guardare il presente, qui e ora, come un processo essenzialmente aperto, in divenire, suscettibile di ricevere forme diverse rispetto a quelle che al momento mostra come proprie, non è che un altro nome per la capacità immaginativa di pensare altrimenti. Capacità tanto più preziosa, anche politicamente, in contesti comunicativi complessi come sono le città, costantemente alle prese con modalità diverse di riconoscimento dell'altro, degli altri.

Processi che riguardano l'architettura, la politica e la società insieme fanno riferimento a uno spazio che prende forma grazie alla presenza di individui, di gruppi, di reti di relazioni: è «uno spazio fisico e relazionale al tempo stesso: un terzo spazio, elaborato ed esperito a partire da rappresentazioni fisiche e percezioni mentali. Uno spazio vissuto» (Maciocco e Tagliagambe 2009: 130 tr. ns.).

È questo lo spazio delle interazioni sociali, dei flussi di comunicazioni, che si alimenta grazie al complesso delle informazioni e delle relazioni fra $\mathrm{i}$ diversi attori sociali.

Se declinato relativamente ai processi comunicativi, può essere interpretato come lo "spazio intermedio" necessario al passaggio o meglio alla traduzione dal linguaggio del mittente a quello del destinatario e viceversa. È uno spazio di soglia, di confine, in cui possono effettivamente verificarsi errori, fraintendimenti, malintesi, ma in cui si definiscono le differenze, i diversi modi di vedere il mondo, i diversi stili di pensiero.

Qui il confine che prende forma, che da linea di demarcazione si fa progressivamente interfaccia, è ciò che Hannah Arendt chiama lo spazio infra (Arendt 1958: 39 tr. it.): lo "spazio intermedio" è in questo senso spazio pubblico, ma anche spazio d'elezione per l'esercizio del progetto e dell'agire politico, perché lì avviene il riconoscimento dell'altro, la traduzione delle esperienze, lì si articola la parola in tutte le sue forme, lì prende corpo il rapporto fra visibile e invisibile. 


\section{Ocula $\mathbf{a}^{13}$}

Architettura e politica: un incrocio di sguardi | Anna Lazzarini, Dare forma allo spazio del convivere.

\section{Ripensare lo spazio pubblico}

La nozione di "spazio pubblico", considerata nelle sue molteplici dimensioni, sembra particolarmente feconda: essa riguarda l'articolazione degli spazi, le funzioni e il significato degli edifici, ma anche la qualità delle relazioni, delle esperienze e della vita urbana che in essi si svolge.

La città è teatro della società: in essa la vita politica è unita a una forma di spazialità, poiché, come parole e azioni scambiate generano lo spazio pubblico - che è spazio in-fra - così anche lo spazio genera forme particolari di socialità e di cittadinanza, configurando buona parte dell'esistenza sociale, politica e culturale. Le società sono sia costituite, sia rappresentate dalle costruzioni e dagli spazi che costruiscono. Evidentemente i cambiamenti che hanno investito la città contemporanea si riflettono sul modo in cui essa riesce a dare forma ai propri spazi pubblici e, ricorsivamente, le trasformazioni sociali, politiche, economiche indotte dalla globalizzazione conferiscono alla dimensione urbana nuove forme, alle pratiche e ai linguaggi della partecipazione politica nuove modalità di espressione, nuovi ambiti di azione.

Lo spazio pubblico non sarà mai uno spazio neutro, poiché rappresenta un punto di convergenza multiplo: storico, formale, linguistico, simbolico, economico, politico. Esso rinvia alla totalità della città, alla specificità della sua esperienza e ne condensa alcuni motivi fondamentali.

Rispetto ai mutamenti in corso, è evidente che qualunque possibilità di ripensare lo spazio pubblico e di riprogettarne le forme non potrà che affrontare la tensione fra logica dei flussi e logica dei luoghi. Di fronte alle rispazializzazioni globali, che prevedono diverse dislocazioni e relazioni fra centri e periferie, il recupero o la reinvenzione di spazi pubblici deve confrontarsi con il fatto che il territorio contiene contemporaneamente nodi locali e reti globali.

La funzione principale della pianificazione diventa quella di ricercare e potenziare al massimo la connettività, sia infra-metropolitana che intermetopolitana, migliorando la capacità dell'area cittadina di operare nello spazio dei flussi. Allo stesso tempo, deve collegare questi nodi anche con lo spazio fisico, connettendo globalità e localismo. Sul piano concreto ciò significa che la pianificazione deve agire su scala cittadina, migliorando l'efficienza dei trasporti, potenziando le forme di decentramento, evitando la segregazione spaziale e opponendosi alla formazione di aree di esclusione e di marginalizzazione. Se lo spazio urbano è oggi contraddistinto dalla multifunzionalità (ben oltre la specializzazione funzionale dell'urbanistica del passato), l'odierna pianificazione procede nel contempo a un decentramento e a un accentramento di individui e di occupazioni, con la formazione di una molteplicità di centri nell'area metropolitana.

Rispetto alle scelte più specificamente architettoniche, nella crisi comunicativa che ha colpito la realtà urbana, il compito fondamentale è quello di recuperare il significato simbolico e culturale dei luoghi. Tuttavia, pur riconoscendo all'architettura un ruolo imprescindibile, è necessario integrare l'approccio architettonico con altri apporti che intendano perseguire una cura socio-spaziale delle forme urbane, che si esplica nel 


\section{Ocula ${ }^{13}$}

Architettura e politica: un incrocio di sguardi | Anna Lazzarini, Dare forma allo spazio del convivere.

tentativo di mettere in relazione località, individui, comunità e flussi globali attraverso la condivisione degli spazi pubblici.

Pianificazione e architettura possono contribuire alla rigenerazione dello spazio pubblico nel nuovo contesto metropolitano, ma solo scelte politiche adeguate e consapevoli della portata e della complessità dei cambiamenti in corso potranno accompagnare i processi che necessitano di apporti molteplici e integrati. È necessario ripensare e valorizzare nuovi spazi comuni di partecipazione, di cittadinanza attiva, di elaborazione collettiva, di traduzione di linguaggi e di culture, di interconnessione, di reticolazione.

Ciò significa favorire una progettualità integrata e complessa, che contempli insieme progettualità architettonica e urbanistica, ma anche sociale, politica, culturale, in grado di sviluppare progetti a partire dalla lettura e dalla risignificazione delle relazioni in gioco nel contesto. Si tratta di una progettazione multidimensionale e integrata in cui gli elementi di riqualificazione urbanistica e architettonica si intrecciano con la cultura, l'economia, l'organizzazione sociale, entro un ripensamento politico complessivo. "Rigenerare" un territorio significa integrare interventi di riqualificazione edilizia e ambientale, azioni sociali e culturali per promuoverne lo sviluppo, attivando processi di partecipazione degli abitanti.

Ciò significa ripensare complessivamente il significato della sfera pubblica e ridefinirne ambiti, linguaggi, azioni, superando l'angustia dello spazio meramente istituzionale per prospettare uno spazio reale e immaginario veramente condiviso. I luoghi pubblici, infatti, in quanto sedi dell'interazione sociale spontanea, sono dispositivi comunicativi fondamentali della società, e le modalità di espressione e comunicazione individuale e collettiva, che accadono oltre le mura delle abitazioni private e oltre i limiti dei circuiti elettronici, e quindi soprattutto nei luoghi pubblici, costituiscono un ambito di grande interesse per la teoria dell'urbanesimo contemporaneo.

Occorre dunque valorizzare un'architettura in grado di produrre simboli condivisi, attraverso l'edificazione di architettoniche significative, che riportino in primo piano l'accezione dello spazio come forma di vita, densità sociale, modalità narrativa e tensione dialogica, recuperando il significato simbolico e culturale dei luoghi. Ciò significa dare forma e senso allo spazio del convivere, entro luoghi aperti e disponibili all'incontro, alla presenza, alla comunicazione: ciò ripropone con intensità "le ragioni dei corpi", per dare vita a spazi e tempi adatti ad accoglierli e a interloquire con loro.

L'intreccio nel tessuto urbano tra flussi, reti, luoghi e corpi prospetta forme rinnovate di essere-in-comune, che invitano architettura, urbanistica, scienze antropologiche, sociali e politiche a farsi interpreti di questa "volontà di connessione", la cifra peculiare per definire le forme spaziali e simboliche, ma anche le stesse modalità di relazione.

Ciò consente di contrastare quelle forme di dissoluzione della città che cominciano a prendere corpo in nome della supremazia della rete infrastrutturale e di una logica di passaggi e di percorsi, incentrata su un'astratta funzionalità, che esaspera tendenze alla frammentarietà e alla debolezza degli incontri e delle relazioni. L'architettura deve invece riaffermare il legame fra spazi privati e pubblici, l’importanza delle soglie 


\section{Ocula ${ }^{13}$}

Architettura e politica: un incrocio di sguardi | Anna Lazzarini, Dare forma allo spazio del convivere.

così come degli elementi di connessione e di mediazione fra spazi diversi, le complesse relazioni dialogiche fra presenze architettoniche, fra vuoti e pieni, e fra gli orditi narrativi delle strade e delle piazze. Teatro fondamentale dell'interazione dialogica, gli spazi aperti pubblici si configurano, in questo senso, come elementi costitutivi e primari della spazialità urbana (Consonni 2000: 106).

Di questa «metafunzione connettiva» (Perulli 2007: 64) la città resta lo spazio privilegiato, smentendo l'idea che l'innovazione della rete sia priva di localizzazione o indifferente al luogo. Naturalmente, compito della politica è quello di perseguire e di valorizzare costantemente tale funzione. Si tratta di accettare la condizione di essere «una terra di mezzo» (Magatti 2005: 41), un "tramite", un "ponte", in grado di garantire forme di policentrismo reticolare. Ciò senza per questo diventare "città senza qualità", ma sapendo mettere in gioco un'identità leggera, multipla, in continua evoluzione e in grado di progettarsi incessantemente, anche se in un processo fragile e faticoso. Anche per tutte queste ragioni le città sono un laboratorio politico di grande interesse, uno spazio dove immaginare e sperimentare forme rinnovate di governo dei processi complessi, che accadono dentro e fuori i loro confini sempre più fluidi.

\section{Bibliografia}

Arendt, $\mathrm{H}$.

1958 The Human Condition, Chicago, Chicago, University of Chicago Press, (tr. it., Vita activa. La condizione umana, Milano, Bompiani, 1976/2009).

Calvino, I.

1972 Le città invisibili, Torino, Einaudi.

Castells, M.

1996 The Rise of the Network Society, The Information Age: Economy, Society and Culture, Cambridge, UK., Blackwell, (tr. it., La nascita della società in rete, 2002).

Castells, M.

1989 The Informational City, Oxford, Blackwell (tr. it., La città delle reti, Venezia, Marsilio, 2004).

Ceruti, M.

1989 La danza che crea. Evoluzione e cognizione nell'epistemologia genetica, Milano, Feltrinelli.

Consonni, G.

2000 Dalla radura alla rete. Inutilità e necessità della città, Milano, Unicopli.

Derrida, J.

1985 "Point de folie: Maintenant l'architecture" in. Tschumi, B., La Case Vide, Paris, La Villette (tr. it., Adesso l'architettura, Milano, Libri Scheiwiller, 2008).

Maciocco, G., Tagliagambe, S.

1997 La città possibile. Territorialità e comunicazione nel progetto urbano, Bari, Dedalo. 


\section{Ocula ${ }^{13}$}

Architettura e politica: un incrocio di sguardi | Anna Lazzarini, Dare forma allo spazio del convivere.

Maciocco, G., Tagliagambe, S.

2009 People and Space. New Forms of Interaction in the City Project, New York, Springer Verlag.

Magatti, M.

2005 "Novum Mediolanum. Logiche di sviluppo e di governo di un nodo globale", in Artoni, Roberto et al., Milano nodo della rete globale. Un itinerario di analisi e proposte, Milano, Bruno Mondadori. 